\title{
A NOTE ON INTEGRAL TRANSFORMS
}

TA LI

To remove the restriction $f_{n}(1)=0$ in my paper [1], one sets

$$
f_{n}(\sigma)=f_{n}(1) \sigma^{-n}+f_{n}^{*}(\sigma)
$$

and

$$
y_{n}(u)=z_{n}(u)+w_{n}(u),
$$

and obtains from

$$
\int_{0}^{1} \frac{T_{n}(u / \sigma) y_{n}(u) d u}{\left(u^{2}-\sigma^{2}\right)^{1 / 2}}=f_{n}(\sigma)
$$

the equations:

$$
\int_{\sigma}^{1} \frac{T_{n}(u / \sigma) z_{n}(u) d u}{\left(u^{2}-\sigma^{2}\right)^{1 / 2}}=f_{n}(1) \sigma^{-n}
$$

and

$$
\int_{0}^{1} \frac{T_{n}(u / \sigma) w_{n}(u) d u}{\left(u^{2}-\sigma^{2}\right)^{1 / 2}}=f_{n}^{*}(\sigma) .
$$

The solution of $(4)$ is found to be

$$
z_{n}(u)=\frac{2 f_{n}(1)}{\pi} \frac{T_{n-1}(u)}{\left(1-u^{2}\right)^{1 / 2}}
$$

by Lemmas 1,2 , and 3, while that of (5) is given in [1] as

$$
w_{n}(u)=-\frac{2}{\pi} \int_{u}^{1} \frac{T_{n-1}(u / v) d\left[v^{n} f_{n}^{*}(v)\right]}{v^{n-1}\left(v^{2}-u^{2}\right)^{1 / 2}} .
$$

Consequently, the solution of (3) is

$$
y_{n}(u)=\frac{2 f_{n}(1)}{\pi} \frac{T_{n-1}(u)}{\left(1-u^{2}\right)^{1 / 2}}-\frac{2}{\pi} \int_{u}^{1} \frac{T_{n-1}(u / v) d\left[v^{n} f_{n}(v)\right]}{v^{n-1}\left(v^{2}-u^{2}\right)^{1 / 2}} .
$$

\section{REFERENCE}

1. Ta Li, A new class of integral transforms, Proc. Amer. Math. Soc. vol. 11 (1960) pp. 290-298.

General Dynamics (Astronautics)

$$
\text { San Diego, California }
$$

Received by the editors September 6, 1960. 